\title{
Serum leptin and adiponectin in overweight and obese postmenopausal women after the six-month weight loss program and their relationship with BMD
}

Yi-Chih Chi ${ }^{\mathrm{a}}$, Pei-Yang Liu , Hyehyung Shin $^{\mathrm{c}}$, Maria Spicer ${ }^{\mathrm{a}}$, Jasminka Z llich $^{\mathrm{a}}$

${ }^{a}$ Department of Nutrition, Food and Exercise Sciences, The Florida State University, ${ }^{b}$ School of Nutrition and Dietetics, University of Akron, ${ }^{C}$ Research Institute of Insurance \& Finance, Samsung Life Insurance

Introduction

The connection between osteoporosis and obesity is becoming a topic of increasing research. The associated cells to these conditions, osteoblasts and adipocytes, originate from bone marrow stromal cells. The adipocyte-secreted hormones, leptin and adiponectin, may be the mediators between adipose tissue and bone. Clinical studies indicate that increased body weight was highly associated with increased BMD and BMC in postmenopausal women. Conversely, a significant decrease in $\mathrm{BMD} / \mathrm{BMC}$ was found in this population during and after weight loss.

\section{Objective}

The aim was to examine the changes in leptin and adiponectin with the weight and body composition change during the six months weight loss program. Additionally, the relationship between two adipokines and BMD of various skeletal sites was also examined.

\section{Method}

Participants were 100 overweight and obese Caucasian postmenopausal women, randomly assigned to three groups after baseline screening. Each group had a hypocaloric diet combined with assigned treatment: placebo, calcium+vitamin D supplements, or low-fat dairy products. Anthropometries, BMD (by iDXA), were measured and serum leptin, adiponectin, and bone markers (osteocalcin, serum NTx and urine CTx) were analyzed with immunoassay kits, at baseline and 6 months. Dietary and activity records were collected, as well. The data were analyzed by SPSS 20.0 (IBM Corporation, Somers, NY), calculating Pearson's and partial correlation coefficients, adjusted for confounders (age or YSM, BMI, physical activity and $\mathrm{Ca}$ and vitamin $\mathrm{D}$ intake). The changes across time were examined using repeated measure analysis of variance.

\section{Results}

Table 1 Principle characteristics and adipokines' levels at baseline and six month $(\mathrm{N}=100)$

Results are expressed as mean \pm SD. BMI, body mass index.

\begin{tabular}{lll} 
& \multicolumn{1}{c}{ Baseline } & \multicolumn{1}{c}{ Six-month } \\
\cline { 2 - 3 } & Mean \pm SD & Mean \pm SD \\
\hline Age (year) & $55.76 \pm 3.69$ & $56.32 \pm 3.70$ \\
Weight $(\mathbf{k g})$ & $82.55 \pm 14.85$ & $79.33 \pm 15.05$ \\
BMI $\left(\mathbf{k g} / \mathbf{m}^{2}\right)$ & $30.86 \pm 5.26$ & $29.62 \pm 5.31$ \\
Fat mass $(\%)$ & $45.33 \pm 4.74$ & $43.80 \pm 5.76$ \\
Years since menopause & $36.46 \pm 20.69$ & $49.97 \pm 4.04$ \\
Leptin $(\mathbf{n g} / \mathbf{m L})$ & $11.83 \pm 5.70$ & $31.70 \pm 21.40$ \\
Adiponectin $(\boldsymbol{\mu g} / \mathbf{m L})$ & $55.76 \pm 3.69$ & $16.42 \pm 8.06$
\end{tabular}

Table 2 Percentage of changes in adipokines, bone, and body composition parameters after six months

\begin{tabular}{|c|c|c|c|}
\hline & \multicolumn{3}{|c|}{ Time effect } \\
\hline & $\%$ change & $\mathbf{F}$ & $\mathbf{p}$ \\
\hline Leptin (ng/mL) & $-12.6 \%$ & $4.83^{*}$ & 0.03 \\
\hline Adiponectin $(\mu \mathrm{g} / \mathrm{mL})$ & $39.1 \%$ & $36.12^{* *}$ & $<0.001$ \\
\hline Total body BMD (g/cm²) & $-0.67 \%$ & $8.12^{*}$ & 0.005 \\
\hline Total femur BMD $\left(\mathrm{g} / \mathrm{cm}^{2}\right)$ & $-0.37 \%$ & 1.74 & 0.19 \\
\hline \multicolumn{4}{|l|}{ Body composition } \\
\hline Fat free mass $(\mathrm{kg})$ & $-1.53 \%$ & $27.05^{* *}$ & $<0.001$ \\
\hline Fat mass (kg) & $-6.7 \%$ & $48.59^{* *}$ & $<0.001$ \\
\hline Total \% body fat & $-3.4 \%$ & $23.76^{\star *}$ & $<0.001$ \\
\hline Body weight (kg) & $-3.9 \%$ & $60.30^{* *}$ & $<0.001$ \\
\hline
\end{tabular}

\section{Serum Leptin}

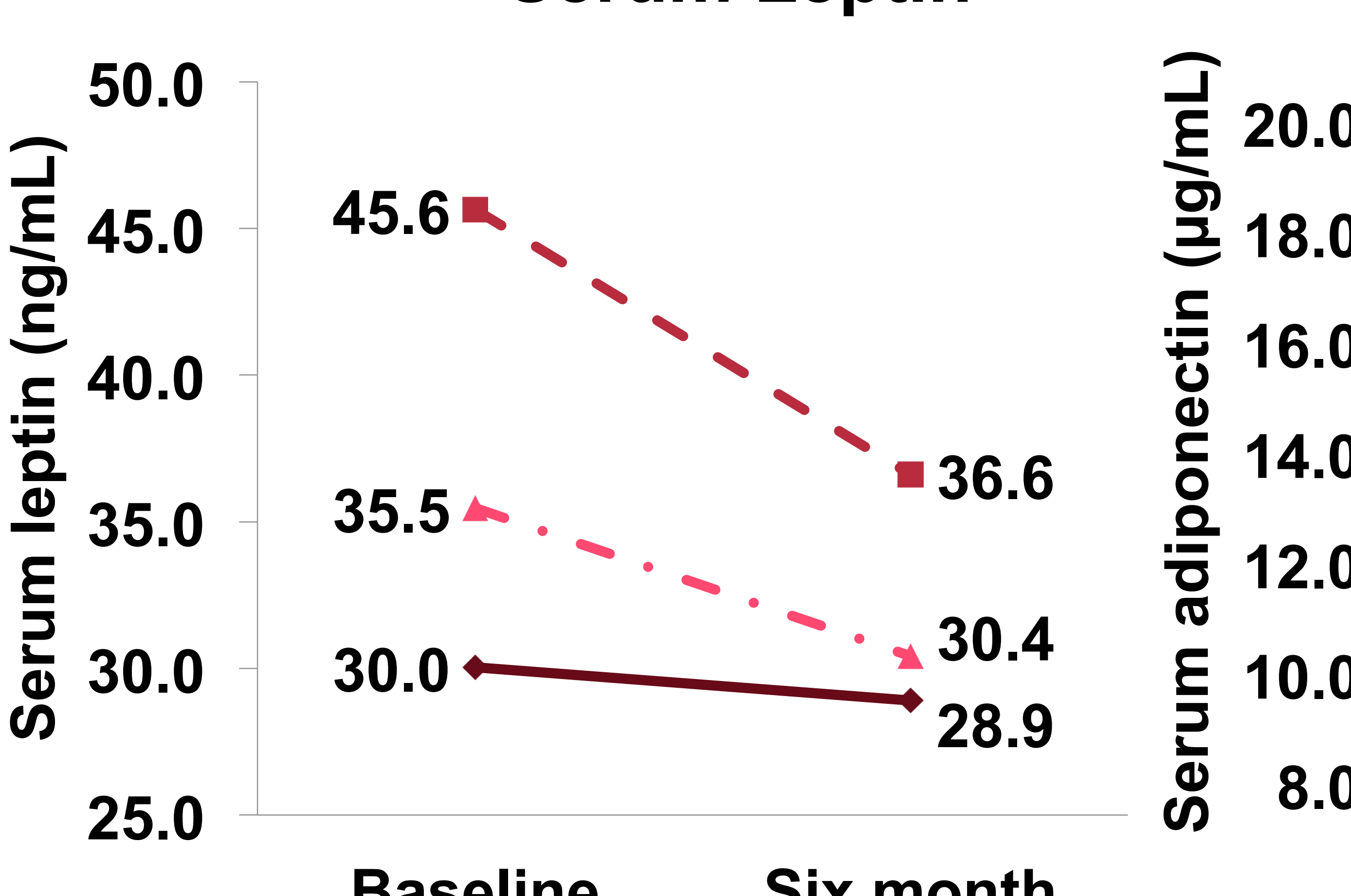

Baseline
Time

Serum Adiponectin

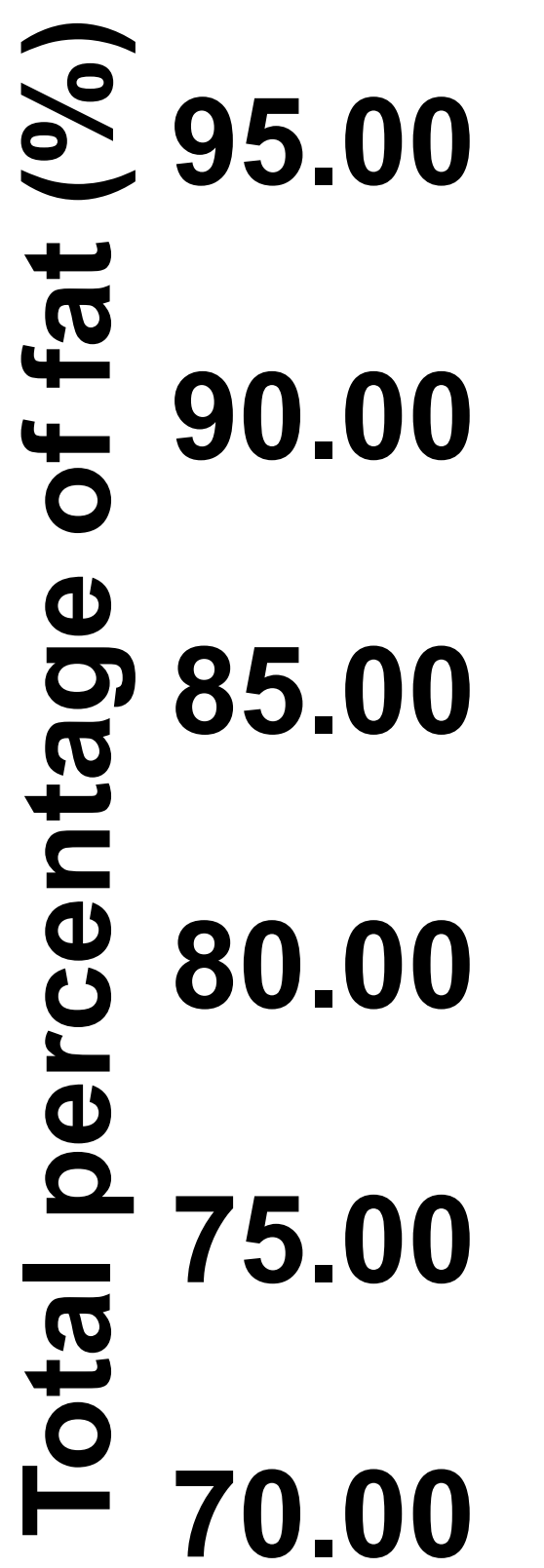

Total percentage of fat

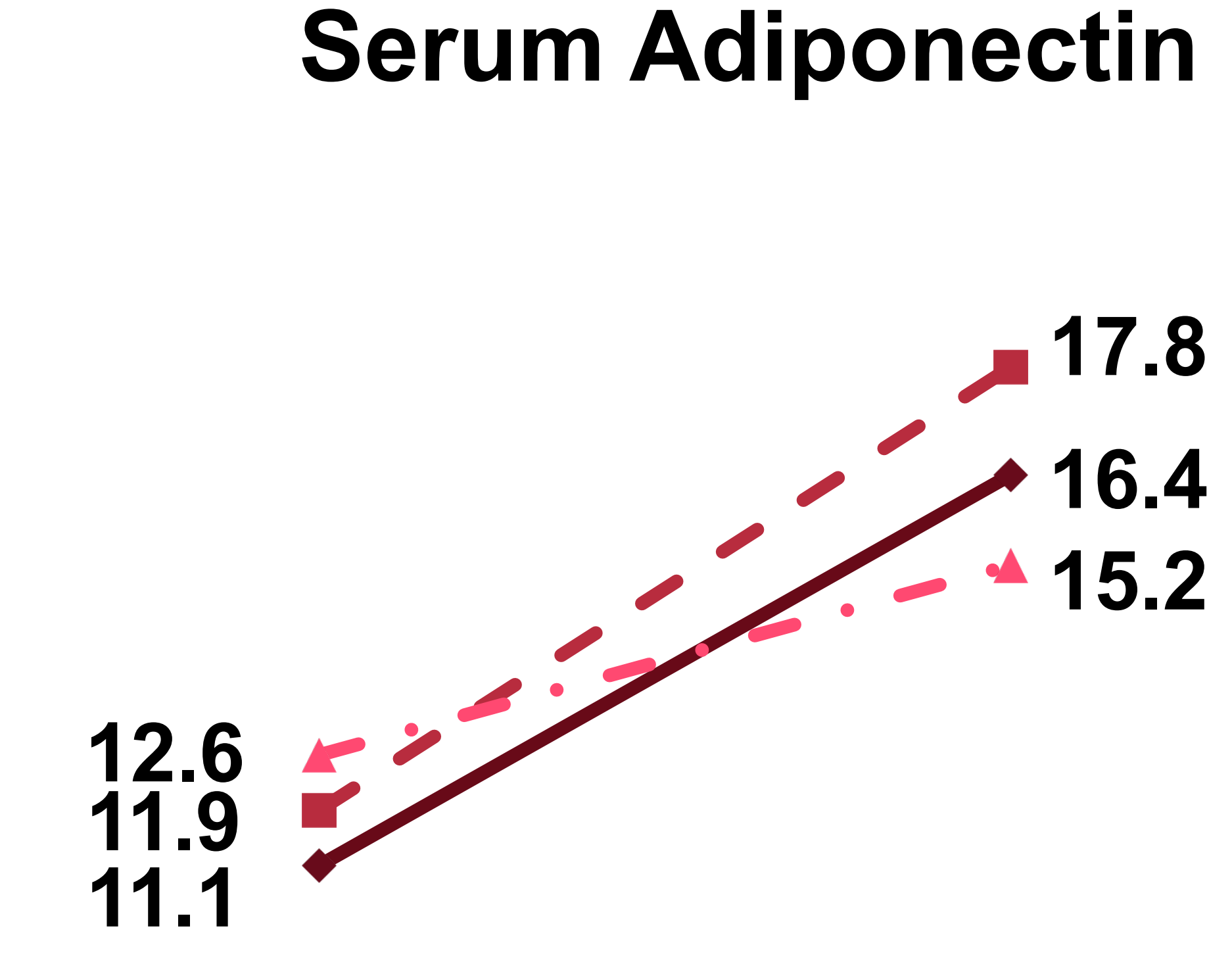

Baseline Six month

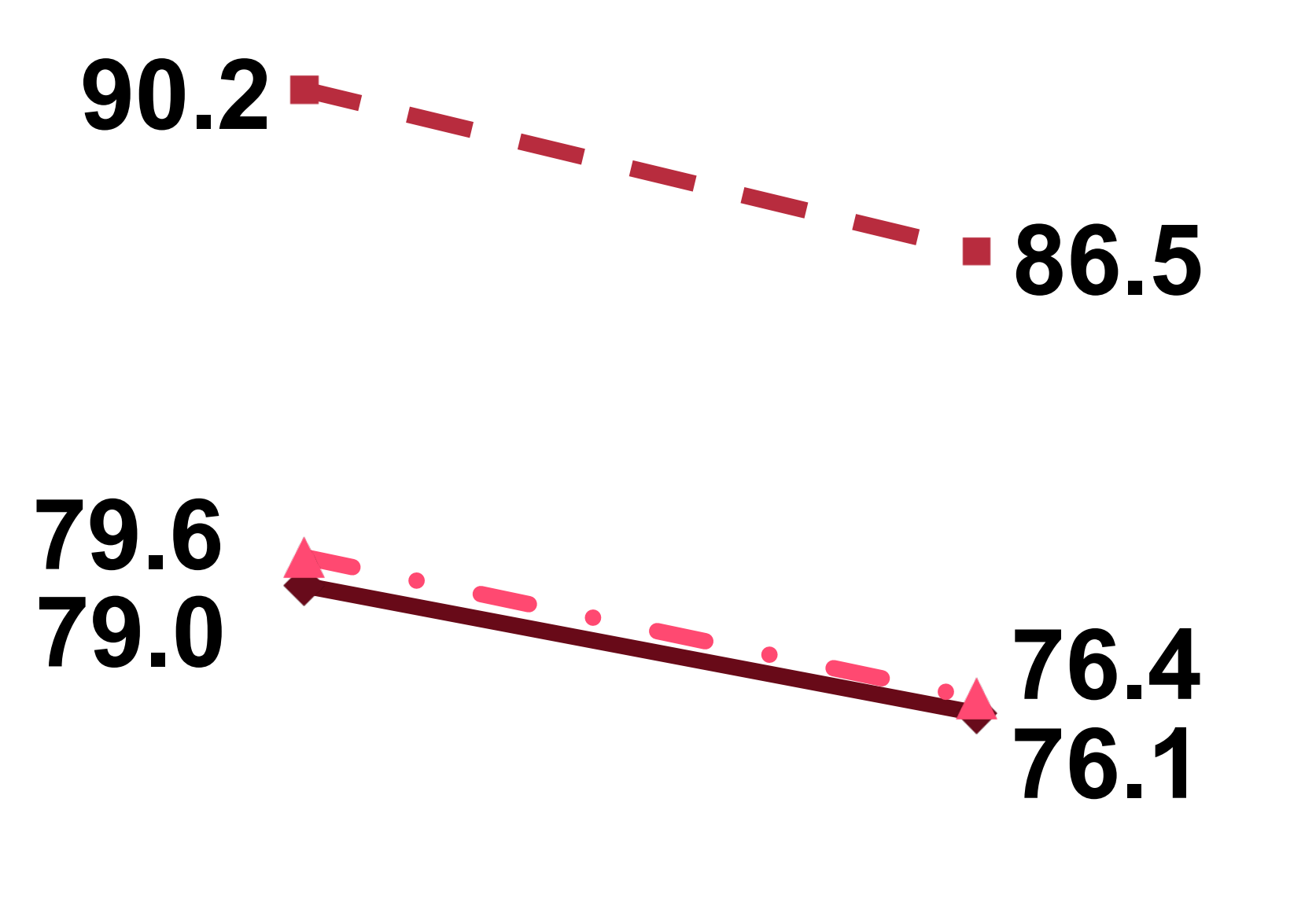

Baseline Six month
- Control group

-Supplement group $\neq$ Dairy group
Table 3 Association between adipokines and bone parameters at baseline using Pearson's and partial correlation

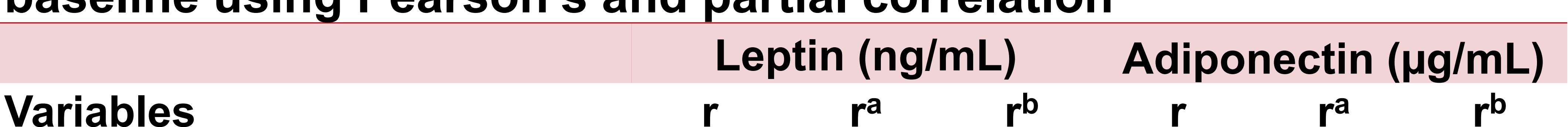

Variables

$\operatorname{BMD}\left(\mathrm{g} / \mathrm{cm}^{2}\right)$

Total body

Total femur

Both forearms

$r \quad r^{a} \quad r^{b}$

Bone markers

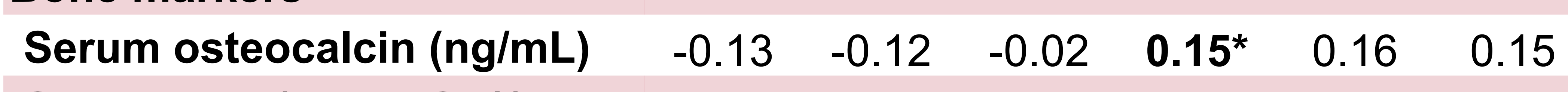

$\begin{array}{lllllll}\text { Serum NTX (nmolBCE/l) } & -0.09 & -0.11 & -0.08 & 0.02 & 0.03 & 0.03\end{array}$

$\begin{array}{lllllll}\text { Urine CTX }(\mu \mathrm{g} / \mathrm{mmol} \cdot \mathrm{Cr}) & -0.04 & -0.03 & -0.01 & 0.03 & 0.04 & 0.03\end{array}$

${ }^{*} P<0.05 . n=184 . B C E$, bone collagen equivalent. $r$ : Unadjusted

$\mathrm{r}^{\mathrm{a}}$ Adjusted for age, physical activity, baseline YSM, dietary calcium and vitamin D intake.

$\mathrm{r}^{\mathrm{b}}$ Adjusted for age, physical activity, baseline YSM, dietary calcium and vitamin $\mathrm{D}$ intake, and BMI.
Table 4 Association between adipokines and bone parameters at 6 month using Pearson's and partial correlation

\begin{tabular}{|c|c|c|c|c|c|c|}
\hline \multirow[b]{2}{*}{ Variables } & \multicolumn{3}{|c|}{ Leptin (ng/mL) } & \multicolumn{3}{|c|}{ Adiponectin $(\mu \mathrm{g} / \mathrm{mL})$} \\
\hline & $\mathbf{r}$ & $\mathbf{r}^{\mathrm{a}}$ & $r^{b}$ & $\mathbf{r}$ & $\mathbf{r}^{\mathbf{a}}$ & $\mathbf{r}^{\mathbf{b}}$ \\
\hline \multicolumn{7}{|l|}{$\mathrm{BMD}\left(\mathrm{g} / \mathrm{cm}^{2}\right)$} \\
\hline Total body & $0.23^{*}$ & 0.19 & 0.09 & -0.02 & -0.04 & -0.05 \\
\hline Total femur & $0.27^{*}$ & $0.21^{*}$ & 0.17 & -0.07 & -0.11 & -0.12 \\
\hline Both forearm & 0.11 & 0.10 & 0.02 & -0.11 & -0.11 & -0.12 \\
\hline \multicolumn{7}{|l|}{ Bone markers } \\
\hline Serum osteocalcin $(\mathrm{ng} / \mathrm{mL})$ & 0.00 & -0.05 & 0.02 & 0.03 & 0.03 & 0.04 \\
\hline Serum NTX (nmolBCE/l) & 0.16 & 0.13 & 0.13 & $-0.25^{*}$ & $-0.27^{*}$ & -0.27 \\
\hline Urine CTX $(\mu \mathrm{g} / \mathrm{mmol} \cdot \mathrm{Cr})$ & 0.06 & 0.04 & 0.05 & -0.02 & -0.02 & -0.02 \\
\hline
\end{tabular}

In conclusion, six-month weight loss resulted in slight bone loss and decreased leptin and increased adiponectin levels. The positive effect of leptin on femoral BMD remained even after its decreased levels caused by weight loss. 